\title{
The Application of Possibility Distribution for Solving Standard Quadratic Optimization Problems
}

\author{
Lunshan $\mathrm{Gao}^{1}$ \\ ${ }^{1}$ School of Engineering and Information Technology, Conestoga College, Kitchener, Ontario, Canada \\ Correspondence: Lunshan Gao, School of Engineering and Information Technology, Conestoga College, \\ Kitchener, Ontario, Canada. E-mail: sgao@conestogac.on.ca; lunshan.gao@gmail.com
}

Received: July 2, 2017

Accepted: July 11, $2017 \quad$ Online Published: July 30, 2017

doi:10.5539/cis.v10n3p60

URL: http://doi.org/10.5539/cis.v10n3p60

\begin{abstract}
A standard quadratic optimization problem (StQP) is to find optimal values of a quadratic form over the standard simplex. The concept of possibility distribution was proposed by L. A. Zadeh. This paper applies the concept of possibility distribution function to solving StQP. The application of possibility distribution function establishes that it encapsulates the constrained conditions of the standard simplex into the possibility distribution function, and the derivative of the StQP formula becomes a linear function. As a result, the computational complexity of StQP problems is reduced, and the solutions of the proposed algorithm are always over the standard simplex. This paper proves that NP-hard StQP problems are in P. Numerical examples demonstrate that StQP problems can be solved by solving a set of linear equations. Comparing with Lagrangian function method, the solutions of the new algorithm are reliable when the symmetric matrix is indefinite.
\end{abstract}

Keywords: standard quadratic optimization, standard simplex, fuzzy number, possibility distribution, P-completeness

\section{Introduction}

StQP problems are widely used in game theory, operating research, system control, financial mathematics, and etc. It is very important branch in applied mathematics and computer science. Note that StQP is NP-hard when the matrix is indefinite (Bomze, 1998, 2002).

Fuzzy sets theory has been applied to quadratic programming. According to Silva, Cruz, Verdegay and Yamakami(Silva et al., 2010), Tang and Wang study quadratic programming problems with a type of fuzzy objective and resource constraints, and it is an interactive approach (Tang \& Wang, 1997); Liu proposed an approach to solve quadratic programming problems with fuzzy costs and fuzzy coefficients in the restrictions set (Liu, 2007); Ammear and Khalifa proposed an approach to solve quadratic programming problems with fuzzy costs, fuzzy matrix coefficients and the restrictions set vector (Ammear \& Khalifa, 2003); Tonon and Bernardini described an approach to solve fuzzy convex programming with fuzzy costs and fuzzy coefficients in restrictions set (Tonon \& Bernardini, 1998).

Bomze studies gradient projection methods for solving StQP problems (Bomze, 1998). He reviewed on traditional gradient projection methods and proposed gradient-like method. Generally speaking, the gradient projection method is to calculate the derivative of the Lagrangian function, and find the Karush-Kuhn-Tucker(KKT) points. The difficulty with traditional gradient projection methods is to obtain a feasible direction via projection onto the feasible constrained conditions of the standard simplex (Bomze, 2002).

Zadeh indicated that" The possibility distribution function associated with a variable is defined to be numerically equal to the membership function of a fuzzy subset" (Zadeh, 1978). He claimed that the membership function of a fuzzy set could serve as a possibility distribution. That is, the membership functions of fuzzy subsets (fuzzy numbers) over a domain can be considered as the possibility distribution of a variable that is defined in the domain.

Drakopoulos proved that if the domain sets are uncountable, the probability measure, possibility measure and the associated fuzzy set system class are equivalent in terms of expressiveness (Drakopoulos, 1995). Drakopoulos also concluded" Over-simplifying a probabilistic model so that its computations can be easily performed may reduce the information conveyed to such a level that possibilities would be preferable not only for their 
simplicity and efficiency advantage but also for their information content."(Drakopoulos, 1995).

Dubios, Foulloy, Mauris and Prade showed that if a fuzzy confidence interval is induced by a probability distribution function(PDF), then the possibility distribution that consists of the fuzzy number is consistent with the PDF (Dubios et al., 2004).

Singpurwalla and Brooker indicated the similarities and differences between membership functions of fuzzy sets and the probability of fuzzy sets, and concluded that the membership function of a fuzzy set is a likelihood function rather than a PDF (Singpurwalla \& Brooker, 2004).

Zadeh presented the definitions of possibility distribution and probability distribution, and denoted the similarity between the possibility distribution and the probability distribution (Zadeh, 2014).

In this paper, instead of using PDFs to describe probability distribution of variables over the standard simplex, possibility distribution functions are used to describe the uncertainty of variables over the standard simplex. Similar to the Lagrangian function, the proposed algorithm calculates the derivative of the quadratic form, and find the KKT points directly. It improves the weakness that the solution of Lagrangian function method is not always over the standard complex (please refer to the example 4 and 5 in section 4). Unlike gradient projection algorithms, the new algorithm does not require iteration for calculating the solutions of StQP problems. As a result, it removes the difficulty that requires to obtain a feasible direction via projection onto the constrained conditions of the standard simplex when gradient projection algorithms are used. This paper also proves that for a given arbitrary possibility distribution, there exists an associated possibility distribution that satisfies the constrained conditions of the standard simplex. This paper is organized as follows. Section 2 describes StQP problems, fuzzy numbers and possibility distributions. Section 3 describes the main results of this paper. Section 4 shows examples. Section 5 discusses the computational complexity of StQP problems. Section 6 is the conclusion.

\section{Preliminary}

\subsection{Notation}

A StQP problem consists of finding optimal values of a quadratic form over the standard simplex. It is described as follows.

$$
\max _{x \in \triangle} f(x)=x^{T} Q x
$$

where $\mathrm{Q}$ is an arbitrary symmetric $n \times n$ matrix; and $\Delta$ is the standard simplex in $\mathrm{n}$-dimensional Euclidean space $R^{n}$, such that,

$$
\Delta=\left\{x \in R^{n} \mid x_{i} \geq 0 ; \sum x_{i}=1\right\}
$$

Note that for a positive definite Q the ellipsoid method (Kozlov et al., 1979) solves the StQP problems in polynomial time. However, if $\mathrm{Q}$ is indefinite, then the StQP is NP-hard. In fact, even if Q has only one negative eigenvalue, the StQP problem is NP-hard (Bomze, 2002).

\subsection{Fuzzy Numbers}

Definition 2.1 A fuzzy number is a fuzzy set which is defined in real number set $R$ (Kaufmann \& Gupta, 1998).

Trapezoidal fuzzy numbers and triangular fuzzy numbers are two representative fuzzy numbers (Kaufmann \& Gupta, 1998). Triangular fuzzy numbers(TFNs) are discussed and used in this paper.

Definition 2.2 A TFN $\tilde{\alpha}$ is denoted as $\tilde{\alpha}=\left(d^{l}, d^{m}, d^{r}\right)$, where $d^{k} \in R(k=l, m, r)$; and $\left(d^{l} \leq d^{m} \leq d^{r}\right) ; d^{m}$ is called the mean value of the TFN $\tilde{\alpha}$.

The membership function $\lambda(t)$ of TFN $\tilde{\alpha}$ is as follows:

$$
\lambda_{\tilde{\alpha}}(t)= \begin{cases}\frac{t-d^{l}}{d^{m}-d^{l}} & t \in \operatorname{supp}\left(\lambda_{\tilde{\alpha}_{L}}(t)\right) \\ \frac{d^{r}-t}{d^{r}-d^{m}} & t \in \operatorname{supp}\left(\lambda_{\tilde{\alpha}_{R}}(t)\right) \\ 0 & t \notin \operatorname{supp}\left(\lambda_{\tilde{\alpha}}(t)\right)\end{cases}
$$

where $\lambda_{\alpha^{\sim} L}(t), \lambda_{\alpha^{`} R}(t)$ is left, right site part of TFN $\tilde{\alpha}$, respectively; $\operatorname{supp}(\lambda(t))$ is the support of function $\lambda(t)$. According to the definition of a TFN $\tilde{\alpha}$, its membership function has the following property.

$$
0 \leq \lambda_{\alpha^{\sim}}(t) \leq 1, t \in R \text {. }
$$


When supp $\lambda(t)=[0,1]$, there are two special TFNs. One is $\tilde{\alpha}_{R}=(0,0,1)$. It is denoted by R-TFN. The other is $\tilde{\alpha}_{L}$ $=(0,1,1)$. It is denoted by L-TFN. The membership functions of R-TFN and L-TFN are as follows.

$$
\lambda_{\tilde{\alpha}_{R}}(t)=\left\{\begin{array}{ll}
1-t, & t \in[0,1] \\
0, & t \notin[0,1],
\end{array}, \lambda_{\tilde{\alpha}_{L}}(t)= \begin{cases}t, & t \in[0,1] \\
0, & t \notin[0,1]\end{cases}\right.
$$

\subsection{Possibility Distribution}

The concept of possibility distribution was proposed by Zadeh (Zadeh, 1978). A possibility distribution function associated with a variable was defined to be numerically equal to the membership function of a fuzzy set.

Serrurier and Prade indicate that a possibility distribution $\pi$ is a mapping from $\Omega$ to $[0,1]$ ( $\Omega$ may be a discrete universe, i.e., $\Omega=\left\{C_{1}, C_{2}, \cdots, C_{q}\right\}$, or a continuous one, i.e. $\Omega=R$ ), and the value $\pi(x)$ is called the possibility degree of the value $\mathrm{x}$ in $\Omega$ (Serrurier \& Prade, 2013). In this paper, the possibility distribution is defined as follows.

Definition 2.3 Let $X \in R^{n}$. Each element $x_{i} \in X$ can be represented with a TFN $\tilde{x}_{i}$ which is characterized by its membership function $\lambda_{x^{\prime} I}(i=1,2, \cdots, n)$. The possibility distribution function associated with the variable $X$ is defined with $\lambda_{X}=\left(\lambda_{x^{r 1}}, \lambda_{x^{2} 2}, \cdots, \lambda_{x^{n} n}\right)$, where $R^{n}$ is a n-dimensional Euclidean space. It is also called triangle possibility distribution due to that their shapes are triangle.

For instance, the Rock-Scissors-Paper game in the game theory, $X=$ (rock, scissors, paper) $\in R^{3}$. Rock, scissors, paper can be described with a TFN $\tilde{\alpha}_{r}, \tilde{\alpha}_{s}, \tilde{\alpha}_{p}$, respectively $\left(\right.$ Gao, 2015). Suppose that $\tilde{\alpha}_{\mathrm{r}}=(0,1 / 4,1)$, $\tilde{\alpha}_{s}=(0,1 / 2,1), \tilde{\alpha}_{\mathrm{p}}=(0,3 / 4,1)$. Then the possibility distribution associate with the variable $X$ is given as $\lambda_{X}=\left(\lambda_{r}, \lambda_{s}\right.$, $\left.\lambda_{p}\right)$. where the membership functions $\lambda_{l}(l=r, s, p)$ of the three TFNs are as follows.

$$
\begin{gathered}
\lambda_{r}(t)=\left\{\begin{array}{lll}
4 t & t \in\left[0, \frac{1}{4}\right] \\
\frac{4}{3}(1-t) & t \in\left[\frac{1}{4}, 1\right] \\
0 & \text { otherwise }
\end{array}, \lambda_{s}(t)= \begin{cases}2 t & t \in\left[0, \frac{1}{2}\right] \\
2(1-t) & t \in\left[\frac{1}{2}, 1\right] \\
0 & \text { otherwise }\end{cases} \right. \\
\lambda_{p}(t)= \begin{cases}\frac{4}{3} t & t \in\left[0, \frac{3}{4}\right] \\
4(1-t) & t \in\left[\frac{3}{4}, 1\right] \\
0 & \text { otherwise }\end{cases}
\end{gathered}
$$

Note that for a given $t \in[0,1]$, a specific possibility distribution is obtained. For example,

$$
\left.\lambda_{X}(t)\right|_{t=\frac{1}{4}}=\left(\frac{1}{\text { rock }}, \frac{0.5}{\text { scissors }}, \frac{0.3}{\text { paper }}\right)=(1,0.5,0.3)
$$

\section{Fundamental Results}

Note that for a finite set $X=\left\{x_{1}, x_{2}, \cdots, x_{n}\right\}$, a probability distribution $p_{X}(x)$ over the set $X$ has the following properties:

$$
\text { (1) } p_{X}\left(x_{i}\right) \geq 0, \forall x_{i} \in X ;(2) \sum_{i=1}^{n} p_{X}\left(x_{i}\right)=1
$$

such that, $p_{X}\left(x_{i}\right)(i=1,2, \cdots, n)$ is over the standard simplex.

$$
\left\{p_{X}(x) \in R^{n} \mid p_{X}\left(x_{i}\right) \geq 0, \sum_{i=1}^{n} p_{X}\left(x_{i}\right)=1\right\}=\triangle
$$

Dubois, Foulloy, Mauris and Prade study probability-possibility transformation theory (Dubois et al., 2004). They indicate that if the probability distribution $p_{X}(x)$ over the set $X$ is denoted by probability values $p=\left(p_{1}\right.$, $\left.p_{2}, \cdots, p_{n}\right)$. Then there exists a triangle possibility distribution $\lambda=\left(\lambda_{1}, \lambda_{2}, \cdots, \lambda_{n}\right)$, and $p$ and $\lambda$ are order-equivalent(Dubois et al., 2004). In this paper, we give the following theorem that indicates that for giving an arbitrary triangle possibility distribution $\lambda$, there exist an associated possibility distribution $\mu$ that is also over the standard simplex, such as $\mu \in \Delta$.

Theorem 3.1 Suppose $\mathrm{X}=\left(\mathrm{x}_{1}, \mathrm{x}_{2}, \cdots, \mathrm{x}_{\mathrm{n}}\right) \in \mathrm{R}^{\mathrm{n}}$. For a given arbitrary triangle possibility distribution $\lambda(\mathrm{t})$ over variable $X$, then there exists an associated possibility distribution $\mu(\mathrm{t})$, and $\mu(\mathrm{t})$ is over the standard simplex, such that $\mu(\mathrm{t}) \in \Delta$. 
Proof. For $X=\left(x_{1}, x_{2}, \cdots, x_{n}\right) \in R^{n}$, one can define a triangle possibility distribution by mapping $x_{i} \in X$ to an arbitrary TFN $\sim \alpha_{i}$. The membership function of $\sim_{i} \alpha_{i}$ is denoted by $\lambda_{\alpha i}(t)$. Based on the property of TFNs, it is clear that $\lambda_{\alpha^{2} i}(t) \in[0,1]$, and $\sum_{i=1}^{n} \lambda_{\tilde{\alpha}_{i}}(t)>0\left(\forall t \in \operatorname{supp}\left(\lambda_{\tilde{\alpha}}(t)\right)\right)$ (we suppose that $\operatorname{supp} \lambda_{\alpha^{i}(i}(t) \cap \operatorname{supp} \lambda_{\alpha^{\prime} i+1}(t) \neq \varnothing(i$

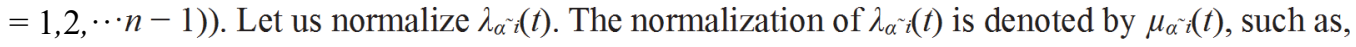

$$
\mu_{\tilde{\alpha}_{i}}(t) \equiv \frac{\lambda_{\tilde{\alpha}_{i}}(t)}{\sum_{k=1}^{n} \lambda_{\tilde{\alpha}_{k}}(t)}
$$

where $t \in \operatorname{supp}\left(\lambda_{\tilde{\alpha}^{\tau} i}(t)\right)$. It is clear that $\mu_{\alpha^{\sim} i}(t) \in[0,1]$ and $\sum_{i=1}^{n} \mu_{\tilde{\alpha}_{i}}(t)=1\left(t \in \operatorname{supp}\left(\mu_{\alpha^{\sim}}(t)\right)\right)$. Thus, for a given arbitrary possibility distribution $\lambda(t)$, there exist an associated possibility distribution $\mu(t)$ that satisfies the following.

$$
\begin{aligned}
\mu(t) \in \Delta=\left\{\mu(t) \in R^{n} \mid \mu_{t}(t) \geq 0 ; \sum \mu_{i}(t)=1\right\} \\
\lambda_{i}(t)\left(\mu_{i}(t)\right) .
\end{aligned}
$$

For simplicity purpose, we consider that $\lambda_{\alpha^{2} i}(t)\left(\mu_{\alpha^{\imath} i}(t)\right)$ is identical to

For each membership function $\lambda_{i}(t)$ of TFN $\left(d^{l}{ }_{i} d^{m}{ }_{i}{ }_{i} d_{i}\right)$, it is not differentiable at the point $t=d^{m}{ }_{i}$. However, $\lambda_{i}(t)$ is differentiable in intervals $\left[d^{l}, d^{m_{i}}\right]$ and $\left[d^{m_{i}}, d^{{ }^{2}}\right]$ according to the definition of TFNs. The following lemma is used for proving Theorem 3.3.

Lemma 3.2 For arbitrary TFNs $\tilde{\alpha}_{i}(i=1,2, \cdots, n)=\left(d_{i}^{1}, d_{i}^{m_{i}}, d_{i}^{r_{i}}\right)$, if their mean values $d^{m_{i}}(i=1,2, \cdots, n)$ satisfy the following.

$$
d_{1}^{m} \leq d_{2}^{m} \leq \cdots \leq d_{n-1}^{m} \leq d_{n}^{m}
$$

then $\forall \lambda_{\mathrm{k}}(\mathrm{t})(\mathrm{k}=1,2, \cdots, \mathrm{n}) \in \lambda(\mathrm{t})=\left\{\lambda_{1}(\mathrm{t}), \lambda_{2}(\mathrm{t}), \cdots, \lambda_{\mathrm{n}}(\mathrm{t})\right\}, \lambda_{\mathrm{k}}(\mathrm{t})$ is a monotonic and continuous function in domain $D_{i}=\left[d_{i-1}^{m}, d_{i}^{m}\right](i=1,2, \cdots, n, n+$

1), where $d_{0}^{m}=d_{1}^{l}$ and $d_{n+1}^{m}=d_{n}^{r}$. The union of all $\mathrm{D}_{\mathrm{i}}$ is denoted by

$$
D=\left[d_{1}^{l}, d_{n}^{r}\right]=\left[d_{1}^{l}, d_{1}^{m}\right] \cup\left[d_{1}^{m}, d_{2}^{m}\right] \cup \cdots \cup\left[d_{n-1}^{m}, d_{n}^{m}\right] \cup\left[d_{n}^{m}, d_{n}^{r}\right] .
$$

Proof. According to the definition of the membership function of TFNs, $\lambda_{k}(t)$ is a continuous function in $D$. Let us use reverse method to prove this lemma.

(1) when $k<i-1$, that means $d_{k}^{m} \leq d_{i-1}^{m}$. Suppose that membership function $\lambda_{k}(t)$ of TFN $\alpha_{k}$ is not monotonic in domain $D_{i}=\left[d_{i-1}^{m}, d_{i}^{m}\right]$. Based on the definition of TFNs, the mean value $d^{m}{ }_{k}$ of TFN $\alpha_{k}$ must be in $D_{i}$, such as $d^{m}{ }_{k} \in D_{i}$. That is,

$$
\lambda\left(d_{k}^{m}\right)=1, d_{i-1}^{m}<d_{k}^{m}<d_{i}^{m}
$$

That contradicts to the fact of $d_{k}^{m} \leq d_{i-1}^{m}$.

(2) when $k$ is either $i-1$ or $i$, that is, either $d_{k}^{m}=d_{i-1}^{m}$ or $d_{k}^{m}=d_{i}^{m}$

Suppose that the membership function $\lambda_{k}(t)$ of TFN $\sim \alpha_{k}$ is not monotonic in domain $D_{i}=\left[d_{i-1}^{m}, d_{i}^{m}\right]$. Based on the definition of TFNs, the mean value $d_{k}^{m}$ of TFN $\sim \alpha_{k}$ must be in $D_{i}$. That means

$$
d_{i-1}^{m}<d_{k}^{m}<d_{i}^{m}
$$

That contradicts to either $d_{k}^{m}=d_{i-1}^{m}$ or $d_{k}^{m}=d_{i}^{m}$.

(3) $k>i$, that means $d^{m}{ }_{k} \geq d^{m}{ }_{i}$. Suppose that the membership function $\lambda_{k}(t)$ of TFN ${ }^{\sim} \alpha_{k}$ is not monotonic in domain $D_{i}=\left[d_{i-1}^{m}, d_{i}^{m}\right]$. According to the property of TFNs, the mean value $d^{m}{ }_{k}$ of TFN $\sim \alpha_{k}$ must be in $D_{i}$, such as $d_{k}^{m} \in D_{i}$. that means

$$
d_{i-1}^{m}<d_{k}^{m}<d_{i}^{m}
$$

That contradicts to the fact of $d_{k}^{m} \geq d_{i-1}^{m}$.

Therefore, the membership function $\lambda_{k}(t)(k=1,2, \cdots, n)$ is monotonic and continuous in domain $D_{i}=\left[d_{i-1}^{m}, d_{i}^{m}\right](i=1,2, \cdots n+1)$.

For StQP problems, $x \in \triangle=\left\{x \in R^{n} \mid x_{i} \geq 0, \sum_{i=1}^{n} x_{i}=1\right\}$. That is,

$0 \leq x_{i} \leq 1(i=1,2, \cdots, n) . x$ is usually described with PDFs (Chen et al. 2013; Ohlson \& Koski, 2012). 
Theorem 3.1 proved that for an arbitrary possibility distribution $\lambda(t)$, there exist an associated possibility distribution $\mu(t)$ that is over the standard simplex. Because both $x$ and $\mu(t)$ are over the standard simplex, $x$ is considered as a probability distribution, $\mu(t)$ is a possibility distribution. The possibility distribution $\mu(t)$ is considered to be equivalent to the probability distribution $x$ by using the probability-possibility transformation theory, such that,

$$
x=\left(x_{1}, x_{2}, \cdots, x_{n}\right) \equiv \mu(t)=\left(\mu_{1}(t), \mu_{2}(t), \cdots, \mu_{n}(t)\right), t \in D_{i}(i=1,2, \cdots, n, n+1)
$$

If one substitutes $x$ in equation (2.1) with $\mu(t)$, equation (2.1) becomes the following

$$
\max _{\mu(t) \in \Delta} f(\mu(t))=(\mu(t))^{T} Q \mu(t) ; t \in D_{i}(i=1,2, \cdots, n, n+1)
$$

where $\mu(t)=\left(\mu_{1}(t), \mu_{2}(t), \cdots, \mu_{n}(t)\right) ; \mu_{i}(t)(i=1,2, \cdots, n)$ is defined by equation (3.1).

Equation (3.3) is considered as an approximation form of StQP (2.1). Instead of solving StQP (2.1), if one can find an optimal solution $t^{*}$ of equation (3.3) in domain $D_{i}(i=1,2, \cdots, n+1)$, then the global optimal solution of equation (3.3) can be found by comparing all the optimal values in each domain. As a result, solution $x^{*}$ can be found by using equation (3.2) and $\mathrm{StQP}(2.1)$ is solved.

Note that in the case of the standard simplex, $D=[0,1]$, and $0 \leq d_{1}^{m} \leq d_{2}^{m} \leq \cdots \cdots \leq d_{n}^{m} \leq 1$. According to Lemma 3.2, $\forall \lambda_{k}(t)\left(\lambda_{k}(t) \in \lambda(t)\right)$, it is monotonic and continue in $D_{i}(i=1,2, \cdots, n, n+1)$. Then one can calculate the derivative of equation (3.3) in domain $D_{i}(i=1,2, \cdots, n, n+1)$, and find KKT points in domain $D_{i}(i=1,2, \cdots$, $n, n+1)$.

Theorem 3.3 StQP (2.1) can be solved by solving $n+1$ number of linear equations.

Proof. Suppose that $d^{m}{ }_{i}$ is the mean value of an arbitrary TFN $\sim \alpha_{i}$, and $0 \leq d_{1}^{m} \leq d_{2}^{m}, \cdots, \leq d_{n}^{m} \leq 1 ; \lambda_{i}(t)$ is the membership function of $\operatorname{TFN} \sim \alpha_{i}$. Note that $\lambda_{i}(t)(i=1,2, \cdots, n)$ is continuous in $[0,1]$ based on the definition of TFNs.

According to Lemma 3.2, we know that $\lambda_{k}(t)(k=1,2, \cdots, n)$ is monotonic and continuous function in domain $D_{i}=\left[d_{i-1}^{m}, d_{i}^{m}\right](i=1,2, \cdots, n, n+1)$. That is, $\lambda_{k}(t)$ is differentiable in domain $D_{i}(i=1,2, \cdots, n, n+1)$. Based on equation (3.1), $\mu_{k}(t)$ is the normalization form of $\lambda_{k}(t)$. Therefore, $\mu_{k}(t)$ is differentiable in domain $D_{i}(i$ $=1,2, \cdots, n, n+1)$ too. Let us calculate the derivative of $f(\mu(t))$ in equation (3.3) regarding $\mathrm{t}$ in domain $D_{i}(i=$ $1,2, \cdots, n, n+1)$. The optimal value of $\operatorname{StQP}(3.3)$ in domain $D_{i}$ satisfies the following equation.

$$
\frac{d f(\mu(t))}{d t}=\left(\frac{d \mu(t)}{d t}\right)^{T} Q \mu(t)+(\mu(t))^{T} Q \frac{d \mu(t)}{d t}=0, t \in D_{i}(i=1, \cdots, n+1)
$$

Since $\lambda_{k}(t)$ is the membership function of an arbitrary TFN, $\lambda_{k}(t)$ is monotonic in domain $D_{i}$, and $\lambda_{k}(t)$ is a linear function of $\mathrm{t}$ in domain $D_{i}$. Thus, $\lambda_{k}(t)$ is denoted by the following a linear function of $t$ in domain $D_{i} . \lambda_{k}(t)=a_{k} t$ $+b_{k}, a_{k} \in R, b_{k} \in R(k=1,2, \cdots, n), t \in D_{i}(i=1,2, \cdots, n+1)$. Then one can rewrite equation (3.1) as follows.

$$
\begin{gathered}
\mu_{k}(t)=\frac{a_{k} t+b_{k}}{\sum_{j=1}^{n}\left(a_{j} t+b_{j}\right)}=\frac{a_{k} t+b_{k}}{\sum_{j=1}^{n} a_{j} t+\sum_{j=1}^{n} b_{j}} \\
(k=1,2, \cdots, n), t \in D_{i}(i=1, \cdots, n+1) .
\end{gathered}
$$

Then the possibility distribution $\mu(t)$ in domain $D_{i}$ becomes the following.

$$
\begin{array}{r}
(\mu(t))^{T}=\left(\frac{a_{1} t+b_{1}}{\sum_{j=1}^{n} a_{j} t+\sum_{j=1}^{n} b_{j}}, \frac{a_{2} t+b_{2}}{\sum_{j=1}^{n} a_{j} t+\sum_{j=1}^{n} b_{j}}, \cdots,\right. \\
\left.\frac{a_{n} t+b_{n}}{\sum_{j=1}^{n} a_{j} t+\sum_{j=1}^{n} b_{j}}\right), t \in D_{i}(i=1, \cdots, n+1)
\end{array}
$$

One can calculate the derivative of $\mu(t)$ regarding $\mathrm{t}$ in domain $D_{i}$ is as follows. 


$$
\begin{array}{r}
\left(\frac{d \mu(t)}{d t}\right)^{T}=\left(\frac{a_{1} \sum_{j=1}^{n} b_{j}-b_{1} \sum_{j=1}^{n} a_{j}}{\left(\sum_{j=1}^{n} a_{j} t+\sum_{j=1}^{n} b_{j}\right)^{2}}, \frac{a_{2} \sum_{j=1}^{n} b_{j}-b_{2} \sum_{j=1}^{n} a_{j}}{\left(\sum_{j=1}^{n} a_{j} t+\sum_{j=1}^{n} b_{j}\right)^{2}}, \cdots,\right. \\
\left.\frac{a_{n} \sum_{j=1}^{n} b_{j}-b_{n} \sum_{j=1}^{n} a_{j}}{\left(\sum_{j=1}^{n} a_{j} t+\sum_{j=1}^{n} b_{j}\right)^{2}}\right), t \in D_{i}(i=1, \cdots, n+1)
\end{array}
$$

If one substitutes $\mu(t)$ and $\frac{d \mu(t)}{d t}$ with equations (3.5) and (3.6), then equation (3.4) becomes the following.

$$
\frac{d f(\mu(t))}{d t}=K(t)\left(V^{T} Q U(t)+(U(t))^{T} Q V\right)=0, t \in D_{i}(i=1,2, \cdots, n, n+1)
$$

where

$$
\begin{gathered}
K(t)=\frac{1}{\left(\sum_{j=1}^{n} a_{j} t+\sum_{j=1}^{n} b_{j}\right)^{2}\left(\sum_{j=1}^{n} a_{j} t+\sum_{j=1}^{n} b_{j}\right)} \\
=\frac{1}{\left(\sum_{j=1}^{n} \lambda_{j}(t)\right)^{3}}, t \in D_{i}(i=1,2, \cdots, n, n+1) \\
V^{T}=\left(a_{1} \sum_{j=1}^{n} b_{j}-b_{1} \sum_{j=1}^{n} a_{j}, a_{2} \sum_{j=1}^{n} b_{j}-b_{2} \sum_{j=1}^{n} a_{j}, \cdots, a_{n} \sum_{j=1}^{n} b_{j}-b_{n} \sum_{j=1}^{n} a_{j}\right) \\
(U(t))^{T}=\left(a_{1} t+b_{1}, a_{2} t+b_{2}, \cdots, a_{n} t+b_{n}\right), t \in D_{i}(i=1,2, \cdots, n, n+1)
\end{gathered}
$$

We suppose that $\left.\operatorname{supp}\left(\lambda_{i}(t)\right) \cap \operatorname{supp}\left(\lambda_{i+1}(t)\right) \neq \emptyset(i=1,2, \cdots n-1)\right)$. Since $\lambda_{i}(t) \in[0,1]$, then $\forall t \in D_{i}, \sum_{j=1}^{n} \lambda_{j}(t)>_{0}$. Then $K(t)$ in equation (3.7) can be ignored. Then, the left hand of equation (3.7) becomes as follows.

$$
V^{T} Q U(t)+(U(t))^{T} Q V=0, t \in D_{i}(i=1,2, \cdots, n, n+1)
$$

$Q$ is a $n \times n$ symmetric matrix, it is easy to prove that equation (3.11) is identical to equation (3.12).

$$
V^{T} Q U(t)=0, t \in D_{i}(i=1,2, \cdots, n, n+1)
$$

When possibility distribution $\lambda(t)$ is given, $a_{i}$ and $b_{i}(i=1,2, \cdots, n)$ are given. Therefore, the elements of $V$ are constant, and the elements of $U(t)$ are linear functions of t. As a result, equation (3.12) becomes a linear equation of $\mathrm{t}$ in domain $D_{i}(i=1,2, \cdots, n, n+1)$. Suppose that equation (3.12) has a solution $t^{*} \in D_{i}$. One can calculate the associated value $x^{*}$ by using equation (3.2), and the optimal value $f\left(x^{*}\right)$. If one repeats the above algorithm for each domain $D_{i}(i=1,2, \cdots, n, n+1)$, which requires to solve $n+1$ linear equation (3.12) in domain $[0,1]$.

In practice, the number of solving linear equations may be reduced by assuming variables $x_{i}(i=k, k+1, \cdots, l)$ have the same possibility distribution. For example, if we suppose that the possibility distributions of all the variables $x_{i}$ are either R-TFN or L-TFN, all the mean values of R-TFNs and L-TFNs take either 0 or 1 , such as $d^{m}{ }_{i} \in\{0,1\}(i=1,2, \cdots, n)$, then $D_{1}=D_{2}=\cdots=D_{n}=D_{n+1}=[0,1]$, and the membership functions of TFNs in possibility distribution are either $\lambda_{\alpha^{\prime} R}$ or $\lambda_{\alpha^{\prime} L}$. One can solve the StQP (2.1) by solving only one linear equation in this case (refer to example 1 and example 2).

The solution of equation (3.7) indicates that function $f(\mu(t))$ in equation (3.3) reaches local optimal value. One needs to know that the local optimal value is a local maximum value or a local minimum value. We have the following theorem.

Theorem 3.4 $S(t)$ is defined as follows.

$$
S(t) \equiv V^{T} Q\left(\frac{d U(t)}{d t}\right), t \in D_{i}(i=1,2, \cdots, n, n+1) .
$$


where $V, U(t)$ is denoted by equation (3.9), equation (3.10), respectively.

Suppose that $t^{*}$ is a valid solution of equation (3.12), such that $\left.\frac{d f(\mu(t))}{d t}\right|_{t=t^{*}}=0$.

If $S\left(t^{*}\right)>0$, then $f\left(\mu\left(t^{*}\right)\right)$ is a local minimum value in domain $D_{i}$. If $S\left(t^{*}\right)<0$, then $f\left(\mu\left(t^{*}\right)\right)$ is a local maximum value in domain $D_{i}$.

Proof. It is clear that function $f(\mu(t))$ is a $R \rightarrow R$ function. The Taylor series of $f(\mu(t))$ at $t^{*}$ is as follows.

$f(\mu(t))-f\left(\mu\left(t^{*}\right)\right)$

$$
\begin{gathered}
=\left.\left(t-t^{*}\right) \frac{d f(\mu(t))}{d t}\right|_{t=t^{*}}+\left.\left(t-t^{*}\right)^{2} \frac{d^{2} f(\mu(t))}{d t^{2}}\right|_{t=t^{*}}+\text { H.O.T. } \\
=\left.\left(t-t^{*}\right)^{2} \frac{d^{2} f(\mu(t))}{d t^{2}}\right|_{t=t^{*}}+\text { H.O.T. }, t \in D_{i}(i=1,2, \cdots, n, n+1) .
\end{gathered}
$$

According to equation (3.7), one knows that

$$
\begin{gathered}
\frac{d f(\mu(t))}{d t}=K(t)\left(V^{T} Q U(t)+(U(t))^{T} Q V=2 K(t) V^{T} Q U(t)\right. \\
t \in D_{i}(i=1,2, \cdots, n+1) .
\end{gathered}
$$

One can obtain the following.

$$
\begin{gathered}
\left.\frac{d^{2} f(\mu(t))}{d t^{2}}\right|_{t=t^{*}}=\left.2 \frac{d K(t)}{d t}\left(V^{T} Q U(t)\right)\right|_{t=t^{*}}+\left.2 K(t)\left(V^{T} Q \frac{d U(t)}{d t}\right)\right|_{t=t^{*}} \\
=\left.2 K(t)\left(V^{T} Q \frac{d U(t)}{d t}\right)\right|_{t=t^{*}}
\end{gathered}
$$

Since equation (3.12) holds at $t=t^{*}$, equation (3.14) becomes as follows.

$$
\begin{array}{r}
f(\mu(t))-f\left(\mu\left(t^{*}\right)\right)=\left.2\left(t-t^{*}\right)^{2} K(t)\left(V^{T} Q \frac{d U(t)}{d t}\right)\right|_{t=t^{*}}+\text { H.O.T. } \\
\quad=2\left(t-t^{*}\right)^{2} K\left(t^{*}\right) S\left(t^{*}\right)+H . O . T ., t \in D_{i}(i=1,2, \cdots, n+1)
\end{array}
$$

Since $K\left(t^{*}\right)>0$ and $\left(t-t^{*}\right)^{2}>0$, therefore, if $S\left(t^{*}\right)>0$, then $f\left(\mu\left(t^{*}\right)\right)$ is a local minimum value; if $S\left(t^{*}\right)<0$, then $f\left(\mu\left(t^{*}\right)\right)$ is a local maximum value.

\subsection{The Algorithm}

Theorem 3.1 indicates that the constrained conditions of variable $x$ being over the standard simplex are encapsulated into the possibility distribution function. This encapsulation technique provides that the users of the algorithm do not need to consider how to make the variable $x$ meet the constrained conditions of StQP problems. According to Theorem 3.1 and Theorem 3.3, the algorithm is described as follows.

Step 1. Find a suitable triangle possibility distribution $\lambda(t)$. The elements of $\lambda(t)$ are membership functions of TFNs, and the mean values of the TFNs are $d^{m}{ }_{i}(i=1,2, \cdots, n)$ and $\left.0 \leq d_{1}^{m} \leq d_{2}^{m} \leq \cdots, \leq d_{n}^{m} \leq 1\right)$. Then the values of coefficients $a_{i} \in R(i=1,2, \cdots, n)$ and $b_{i} \in R(i=1,2, \cdots, n)$ in equation (3.5) and equation (3.6) are known.

Step 2. Determine $V$ and $U(t)$ in domain $D_{i}$. One can calculate $V$ and $U(t)$ based on equation (3.9) and equation (3.10) because $a_{i}(i=1,2, \cdots, n)$ and $b_{i}(i=1,2, \cdots, n)$ are given.

Step 3. Find solution $t^{*}$ in domain $D_{i}$. Solve the linear equation (3.12) in domain $D_{i}$, and find a solution $t^{*}$.

Step 4. Verify the solution $t^{*}$. If $t^{*} \in D_{i}$, then go to Step 5; if $t^{*} \notin D_{i}$ or equation (3.12) does not have solution in $D_{i}$, then ignore the $t^{*}$ and go to Step 2 and repeat the steps for next domain $D_{i+1}$.

Step 5. Calculate $x^{*}$ and $f\left(x^{*}\right)$. Find the solution $x^{*}=\mu\left(t^{*}\right)$ in domain $D_{i}$ with equation (3.5), and compute $f\left(x^{*}\right)$.

Step 6. Determine that $f\left(x^{*}\right)$ is a local minimum value or a local maximum value. Calculate $S\left(t^{*}\right)$ in equation (3.13), and decide $f\left(x^{*}\right)$ is a local minimum value or local maximum value based on Theorem 3.4.

Step 7. Find the global maximum(minimum) solutions. Compare all the local maximum(minimum) values of $f\left(x^{*}\right)$ that are found in each domain, find the solution $x^{*}$ of the global maximum(minimum) value. 


\section{Examples}

Example 1. Solve the following StQP problem.

$$
\max _{\mathrm{x} \in \Delta} f(x)=x^{T} Q x
$$

where $x \in R^{3}$ and $\Delta$ is the standard simplex, and $Q$ is defined as follows.

$$
Q=\left(\begin{array}{lll}
0 & 1 & 2 \\
1 & 0 & 1 \\
2 & 1 & 0
\end{array}\right)
$$

Note that symmetric matrix $Q$ has two negative eigenvalues and one positive eigenvalue. $Q$ is an indefinite matrix.

Step 1. A triangle possibility distribution is given by the following three TFNs in $D=[0,1]$.

$$
(0,1,1), \quad(0,0,1), \quad(0,1,1) .
$$

The membership functions of the TFNs are as follows.

$$
\lambda_{1}(t)=t(t \in[0,1]), \lambda_{2}(t)=1-t(t \in[0,1]), \lambda_{3}(t)=t(t \in[0,1]) .
$$

The mean values of the three TFNs are $d_{1}^{m}=1, d_{2}^{m}=0$, and $d_{3}^{m}=1$. Thus,

$$
D_{1}=D_{2}=D_{3}=[0,1] \text {. }
$$

Note that the values of $a_{i}(i=1,2,3)$ and $b_{i}(i=1,2,3)$ are as follows in this case.

$$
a_{1}=1, b_{1}=0 ; a_{2}=-1, b_{2}=1 ; a_{3}=1, b_{3}=0 .
$$

Step 2. Calculate $V$ and $U(t)$. Based on equation (3.9) and equation (3.10), one calculates $V$ and $U(t)$ as follows.

$$
V^{T}=(1,-2,1),(U(t))^{T}=(t, 1-t, t) .
$$

Step 3. Solve the equation (3.12).

$$
(1,-2,1)\left(\begin{array}{lll}
0 & 1 & 2 \\
1 & 0 & 1 \\
2 & 1 & 0
\end{array}\right)\left(\begin{array}{c}
t \\
1-t \\
t
\end{array}\right)=0
$$

The solution of the above equation is $t^{*}=1$.

Step 4 . Verify the value of $t^{*}$. Since $t^{*} \in[0,1]$, the value is valid.

Step 5. Find the solution $x^{*} . x^{*}$ is obtained from equation (3.5).

$$
x^{*}=\left.\left(\frac{t}{1+t}, \frac{1-t}{1+t}, \frac{t}{1+t}\right)\right|_{t^{*}=1}=\left(\frac{1}{2}, 0, \frac{1}{2}\right)
$$

Step 6. Determine $f\left(x^{*}\right)$ is a local maximum(minimum) value. One can calculate $S(t)$ in equation (3.13) as follows.

$$
S(t)=(1,-2,1)\left(\begin{array}{lll}
0 & 1 & 2 \\
1 & 0 & 1 \\
2 & 1 & 0
\end{array}\right)\left(\begin{array}{c}
1 \\
-1 \\
1
\end{array}\right)=-2<0
$$

therefore, $f\left(x^{*}\right)=1$ is a local maximum value.

Step 7. Find the global maximum value. $f(x)$ has a maximum value 1 at $x^{*}=\left(\frac{1}{2}, 0, \frac{1}{2}\right)$.

Note that one can find the same value by using Lagrangian function method.

Example 2. Solve the following StQP problem

$$
\max _{x \in \triangle} f(x)=x^{T} Q x
$$

where $x \in R^{4}, \Delta$ is the standard simplex, and $Q$ is as follows. 


$$
Q=\left(\begin{array}{llll}
0 & 1 & 1 & 1 \\
1 & 0 & 1 & 1 \\
1 & 1 & 0 & 1 \\
1 & 1 & 1 & 0
\end{array}\right)
$$

The symmetric matrix $Q$ is indefinite because it has one positive eigenvalue and three negative eigenvalues.

Step 1. A triangle possibility distribution is given by the following four TFNs.

$$
(0,0,1), \quad(0,1,1), \quad(0,0,1), \quad(0,1,1)
$$

the associated membership functions of the TFNs are

$$
\begin{aligned}
& \lambda_{1}(t)=-t+1(t \in[0,1]), \lambda_{2}(t)=t(t \in[0,1]), \\
& \lambda_{3}(t)=-t+1(t \in[0,1]), \lambda_{4}(t)=t(t \in[0,1]) .
\end{aligned}
$$

Note that the mean values of the TFNs are $d_{1}^{m}=d_{3}^{m}=0$, and $d_{2}^{m}=d_{4}^{m}=1$. Then domains are as follows. $D_{1}$ $=D_{2}=D_{3}=D_{4}=[0,1]$.

Note that the values of $a_{i}(i=1,2,3,4)$ and $b_{i}(i=1,2,3,4)$ are as follows in this case.

$$
a_{1}=-1, b_{1}=1, a_{2}=1, b_{2}=0, a_{3}=-1, b_{3}=1, a_{4}=1, b_{4}=0 .
$$

Step 2. Calculate $V$ and $U(t)$. Based on equation (3.9) and equation (3.10), one can obtain $V$ and $U(t)$ as follows.

$$
V^{T}=(-2,2,-2,2) ; \quad(U(t))^{T}=(-t+1, t,-t+1, t) .
$$

Step 3. Solve the equation (3.12).

$$
(-2,2,-2,2)\left(\begin{array}{llll}
0 & 1 & 1 & 1 \\
1 & 0 & 1 & 1 \\
1 & 1 & 0 & 1 \\
1 & 1 & 1 & 0
\end{array}\right)\left(\begin{array}{c}
-t+1 \\
t \\
-t+1 \\
t
\end{array}\right)=0
$$

The solution of above equation is $t^{*}=\frac{1}{2}$.

Step 4. Verify the solution $t^{*}$. Note that $t^{*}=\frac{1}{2} \in[0,1]$. Therefore, the solution $t^{*}=\frac{1}{2}$ is valid.

Step 5. Find the solution $x^{*}$ and $f\left(x^{*}\right)$. Find the solution $x^{*}$ by using equation (3.5).

$$
x^{*}=\left.\left(\frac{-t+1}{2}, \frac{t}{2}, \frac{-t+1}{2}, \frac{t}{2}\right)\right|_{t=\frac{1}{2}}=\left(\frac{1}{4}, \frac{1}{4}, \frac{1}{4}, \frac{1}{4}\right) \text {, and } f\left(x^{*}\right)=\frac{3}{4}=0.75
$$

Step 6. Determine that $f\left(x^{*}\right)$ is local maximum(minimum) value. One can calculate $S(t)$ in equation (3.13) as follows.

$$
S(t)=(-2,2,-2,2)\left(\begin{array}{llll}
0 & 1 & 1 & 1 \\
1 & 0 & 1 & 1 \\
1 & 1 & 0 & 1 \\
1 & 1 & 1 & 0
\end{array}\right)\left(\begin{array}{l}
-1 \\
1 \\
-1 \\
1
\end{array}\right)=-8<0
$$

therefore, $f\left(x^{*}\right)=0.75$ is a local maximum value.

Step 7. Find the global maximum value. $f(x)$ has the maximum value 0.75 at $x^{x^{*}}=\left(\frac{1}{4}, \frac{1}{4}, \frac{1}{4}, \frac{1}{4}\right)$.

Note that one can find the same value by using Lagrangian function method.

Example 3. Solve the following StQP problem

$$
\operatorname{maxf}_{\mathrm{x} \in \Delta}(\mathrm{x})=\mathrm{x}^{\mathrm{T}} \mathrm{Qx}
$$

where $x \in R^{3}, \Delta$ is the standard simplex, and $Q$ is as follows. 


$$
Q=\left(\begin{array}{ccc}
1 & 1 & -1 \\
1 & 1 & 3 \\
-1 & 3 & 1
\end{array}\right)
$$

The symmetric matrix $Q$ is indefinite because it has one negative eigenvalue and two positive eigenvalues.

A triangle possibility distribution is given by the following three TFNs in $D=[0,1]$.

$$
(0,0,1), \quad\left(0, \frac{1}{2}, 1\right), \quad(0,1,1)
$$

The membership functions of TFNs are as follows.

$$
\lambda_{1}(t)=-t+1(t \in[0,1]), \quad \lambda_{2}(t)=\left\{\begin{array}{ll}
2 t & t \in\left[0, \frac{1}{2}\right] \\
2-2 t & t \in\left[\frac{1}{2}, 1\right]
\end{array}, \quad \lambda_{3}(t)=t(t \in[0,1]) .\right.
$$

The mean values are $d_{1}^{m}=0, d_{2}^{m}=\frac{1}{2}, d_{3}^{m}=1$, which divides $D=[0,1]$ into two domains, such as $D_{1}=\left[0, \frac{1}{2}\right]$ and $D_{2}=\left[\frac{1}{2}, 1\right]$. As mentioned in section (3.1), let us solve the StQP in each domain.

(1) $D_{1}=\left[0, \frac{1}{2}\right]$.

Step 1 . Note that the coefficients $a_{i}(i=1,2,3)$ and $b_{i}(i=1,2,3)$ are as follows in this case.

$$
a_{1}=-1, \quad b_{1}=1 ; \quad a_{2}=2, \quad b_{2}=0 ; \quad a_{3}=1, \quad b_{3}=0 .
$$

Step 2. Calculate $V$ and $U(t)$. Based on equation (3.9) and equation (3.10), one can calculate $V$ and $U(t)$ as follows.

$$
\mathrm{V}^{\mathrm{T}}=(-3,2,1) ;(\mathrm{U}(\mathrm{t}))^{\mathrm{T}}=(-\mathrm{t}+1,2 \mathrm{t}, \mathrm{t})
$$

Step 3. Solve the equation (3.12).

$$
(-3,2,1)\left(\begin{array}{ccc}
1 & 1 & -1 \\
1 & 1 & 3 \\
-1 & 3 & 1
\end{array}\right)\left(\begin{array}{c}
-t+1 \\
2 t \\
t
\end{array}\right)=0
$$

The solution of above equation is $t^{*}=\frac{1}{8}$.

Step 4 . Verify the value of $t^{*}$. Since $t^{*} \in\left[0, \frac{1}{2}\right]$, the value is valid.

Step 5. Find the values of $x^{*}$ and $f\left(x^{*}\right)$. The value of $x^{*}$ is obtained from equation (3.5).

$$
x^{*}=\left.\left(\frac{1-t}{1+2 t}, \frac{2 t}{1+2 t}, \frac{t}{1+2 t}\right)\right|_{t=\frac{1}{8}}=\left(\frac{7}{10}, \frac{1}{5}, \frac{1}{10}\right) \text {. and } f\left(x^{*}\right)=\frac{4}{5}=0.8 \text {. }
$$

Step 6. Determine $f\left(x^{*}\right)$ is a local maximum or minimum value. One can calculate $S(t)$ in equation (3.13) as follows.

$$
S(t)=(-3,2,1)\left(\begin{array}{ccc}
1 & 1 & -1 \\
1 & 1 & 3 \\
-1 & 3 & 1
\end{array}\right)\left(\begin{array}{c}
-1 \\
2 \\
1
\end{array}\right)=16>0
$$

based on Theorem 3.4, $f\left(x^{*}\right)=\frac{4}{5}=0.8$ is a local minimum value.

(2) $D_{2}=\left[\frac{1}{2}, 1\right]$

Step 1 . Note that the coefficients $a_{i}(i=1,2,3)$ and $b_{i}(i=1,2,3)$ are as follows in this case.

$$
a_{1}=-1, \quad b_{1}=1 ; \quad a_{2}=-2, \quad b_{2}=2 ; \quad a_{3}=1, \quad b_{3}=0 .
$$

Step 2. Calculate $V$ and $U(t)$. Based on equation (3.9) and equation (3.10), one can calculate $V$ and $U(t)$ as follows. 


$$
V^{T}=(-1,-2,3) ; \quad(U(t))^{T}=(-t+1,-2 t+2, t) .
$$

Step 3. Solve the equation (3.12).

$$
(-1,-2,3)\left(\begin{array}{ccc}
1 & 1 & -1 \\
1 & 1 & 3 \\
-1 & 3 & 1
\end{array}\right)\left(\begin{array}{c}
-t+1 \\
-2 t+2 \\
t
\end{array}\right)=0
$$

The solution of above equation is $t^{*}=\frac{3}{4}$.

Step 4. Verify the value of $t^{*}$. Since $t^{*} \in\left[\frac{1}{2}, 1\right]$, the value is valid.

Step 5. Find the values of $x^{*}$ and $f\left(x^{*}\right)$. The value of $x^{*}$ is obtained from equation (3.5).

$$
x^{*}=\left.\left(\frac{1-t}{3-2 t}, \frac{-2 t+2}{3-2 t}, \frac{t}{3-2 t}\right)\right|_{t=\frac{3}{4}}=\left(\frac{1}{6}, \frac{1}{3}, \frac{1}{2}\right) \cdot \operatorname{and} f\left(x^{*}\right)=\frac{4}{3} .
$$

Step 6. Determine $f\left(x^{*}\right)$ is a local maximum or minimum value. One can calculate $S(t)$ in equation (3.13) as follows.

$$
S(t)=(-1,-2,3)\left(\begin{array}{ccc}
1 & 1 & -1 \\
1 & 1 & 3 \\
-1 & 3 & 1
\end{array}\right)\left(\begin{array}{c}
-1 \\
-2 \\
1
\end{array}\right)=-8<0
$$

Based on Theorem 3.4, $f\left(x^{*}\right)=\frac{4}{3}$ is a local maximum value.

Step 7. Find the global maximum(minimum) value. Combining case (1) and

(2) together, one can conclude that $f(x)$ has the minimum value $f\left(x^{*}\right)=\frac{4}{5}$

at $x^{*}=\left(\frac{7}{10}, \frac{1}{5}, \frac{1}{10}\right)$, and has the maximum value $f\left(x^{*}\right)=\frac{4}{3}$ at $x^{*}=\left(\frac{1}{6}, \frac{1}{3}, \frac{1}{2}\right)$.

Note that one can find a solution $x^{*}=\left(\frac{1}{2}, \frac{1}{2}, 0\right)$ by using Lagrangian function method. $f\left(x^{*}\right)=1$ at $x^{*}=\left(\frac{1}{2}, \frac{1}{2}, 0\right)$. Comparing with the solutions that are found with the new algorithm, the value $f\left(x^{*}\right)=1$ at $x^{*}=\left(\frac{1}{2}, \frac{1}{2}, 0\right)$ that is found by using Lagrangian function method is neither a maximum value nor a minimum value of the StQP. That indicates that the proposed algorithm is efficient and effective.

Example 4. Solve the following StQP problem

$$
\min _{\mathrm{x} \in \Delta} f(x)=x^{T} Q x
$$

where $x \in R^{5}, \Delta$ is the standard simplex, and $Q$ is as follows.

$$
Q=\left(\begin{array}{ccccc}
1 & -1 & 1 & 2 & -3 \\
-1 & 2 & -3 & -3 & 4 \\
1 & -3 & 5 & 6 & -4 \\
2 & -3 & 6 & 5 & -8 \\
-3 & 4 & -4 & -8 & 16
\end{array}\right)
$$

The symmetric matrix $Q$ is indefinite because it has exactly one negative eigenvalue, and $Q$ is copositive (Bolor, 2013).

A triangle possibility distribution is given by the following five TFNs in $D=[0,1]$. 


$$
(0,0,1),\left(0, \frac{1}{4}, 1\right),\left(0, \frac{1}{2}, 1\right),\left(0, \frac{3}{4}, 1\right),(0,1,1)
$$

The membership functions of TFNs are as follows.

$$
\begin{gathered}
\lambda_{1}(t)=-t+1(t \in[0,1]), \quad \lambda_{2}(t)=\left\{\begin{array}{ll}
4 t & t \in\left[0, \frac{1}{4}\right] \\
\frac{4}{3}(1-t) & t \in\left[\frac{1}{4}, 1\right]
\end{array},\right. \\
\lambda_{3}(t)=\left\{\begin{array}{ll}
2 t & t \in\left[0, \frac{1}{2}\right] \\
2(1-t) & t \in\left[\frac{1}{2}, 1\right]
\end{array}, \lambda_{4}(t)=\left\{\begin{array}{ll}
\frac{4}{3} t & t \in\left[0, \frac{3}{4}\right] \\
4(1-t) & t \in\left[\frac{3}{4}, 1\right]
\end{array}, \lambda_{5}(t)=t(t \in[0,1])\right.\right.
\end{gathered}
$$

The mean values are $d_{1}^{m}=0, d_{2}^{m}=\frac{1}{4}, d_{3}^{m}=\frac{1}{2}, d_{4}^{m}=\frac{3}{4}, d_{5}^{m}=1$, which divides $D=[0,1]$ into four domains, such as $D_{1}=\left[0, \frac{1}{4}\right], D_{2}=\left[\frac{1}{4}, \frac{1}{2}\right], D_{3}=\left[\frac{1}{2}, \frac{3}{4}\right]$ and $D_{2}=\left[\frac{3}{4}, 1\right]$. As mentioned in section (3.1), let us solve the StQP in each domain.

(1) $D_{1}=\left[0, \frac{1}{4}\right]$.

Step 1 . Note that the coefficients $a_{i}$ and $b_{i}$ are as follows in this case.

$$
a_{1}=-1, b_{1}=1 ; a_{2}=4, b_{2}=0 ; a_{3}=2, b_{3}=0 ; a_{4}=\frac{4}{3}, b_{4}=0 ; a_{5}=1, b_{5}=0
$$

Step 2. Calculate $V$ and $U(t)$. Based on equation (3.9) and equation (3.10), one can calculate $V$ and $U(t)$ as follows.

$$
V^{T}=\left(-\frac{25}{3}, 4,2, \frac{4}{3}, 1\right) ; \quad(U(t))^{T}=\left(-t+1,4 t, 2 t, \frac{4}{3} t, t\right) .
$$

Step 3. Solve the equation (3.12).

$$
\left(-\frac{25}{3}, 4,2, \frac{4}{3}, 1\right)\left(\begin{array}{ccccc}
1 & -1 & 1 & 2 & -3 \\
-1 & 2 & -3 & -3 & 4 \\
1 & -3 & 5 & 6 & -4 \\
2 & -3 & 6 & 5 & -8 \\
-3 & 4 & -4 & -8 & 16
\end{array}\right)\left(\begin{array}{c}
-t+1 \\
4 t \\
2 t \\
\frac{4}{3} t \\
t
\end{array}\right)=0
$$

The solution of above equation is $t^{*}=\frac{32}{161} \approx 0.198$.

Step 4 . Verify the value of $t^{*}$. Since $t^{*} \in\left[0, \frac{1}{4}\right]$, the value is valid.

Step 5. Find the values of $x^{*}$ and $f\left(x^{*}\right)$. The value of $x^{*}$ is obtained from equation (3.5).

$$
\begin{gathered}
x^{*}=\left.\left(\frac{3(1-t)}{3+22 t}, \frac{12 t}{3+22 t}, \frac{6 t}{3+22 t}, \frac{4 t}{3+22 t}, \frac{3 t}{3+22 t}\right)\right|_{t=\frac{32}{161}} \\
=\left(\frac{387}{1187}, \frac{384}{1187}, \frac{192}{1187}, \frac{128}{1187}, \frac{96}{1187}\right) \text {.and } f\left(x^{*}\right)=\frac{193481}{1408969} \approx 0.137 .
\end{gathered}
$$

Step 6. Determine $f\left(x^{*}\right)$ is a local maximum or minimum value. One can calculate $S(t)$ in equation (3.13) as follows.

$$
S=\left(-\frac{25}{3}, 4,2, \frac{4}{3}, 1\right)\left(\begin{array}{ccccc}
1 & -1 & 1 & 2 & 3 \\
-1 & 2 & -3 & -3 & 4 \\
1 & -3 & 5 & 6 & -4 \\
2 & -3 & 6 & 5 & -8 \\
-3 & 4 & -4 & -8 & 16
\end{array}\right)\left(\begin{array}{c}
-1 \\
4 \\
2 \\
\frac{4}{3} \\
1
\end{array}\right)=\frac{161}{3}>0
$$

based on Theorem 3.4, $f\left(x^{*}\right)=\frac{193481}{1408969} \approx 0 \cdot 137$ is a local minimum value. 
(2) $D_{2}=\left[\frac{1}{4}, \frac{1}{2}\right]$.

Step 1. Note that the coefficients $a_{i}$ and $b_{i}$ are as follows in this case.

$$
a_{1}=-1, b_{1}=1 ; a_{2}=-\frac{4}{3}, b_{2}=\frac{4}{3} ; a_{3}=2, b_{3}=0 ; a_{4}=\frac{4}{3}, b_{4}=0 ; a_{5}=1, b_{5}=0
$$

Step 2. Calculate $V$ and $U(t)$. Based on equation (3.9) and equation (3.10), one can calculate $V$ and $U(t)$ as follows.

$$
V^{T}=\left(-\frac{13}{3},-\frac{52}{9}, \frac{14}{3}, \frac{28}{9}, \frac{7}{3}\right) ; \quad(U(t))^{T}=\left(-t+1, \frac{4}{3}(1-t), 2 t, \frac{4}{3} t, t\right)
$$

Step 3. Solve the equation (3.12).

$$
\left(-\frac{13}{3},-\frac{52}{9}, \frac{14}{3}, \frac{28}{9}, \frac{7}{3}\right)\left(\begin{array}{ccccc}
1 & -1 & 1 & 2 & -3 \\
-1 & 2 & -3 & -3 & 4 \\
1 & -3 & 5 & 6 & -4 \\
2 & -3 & 6 & 5 & -8 \\
-3 & 4 & -4 & -8 & 16
\end{array}\right)\left(\begin{array}{c}
-t+1 \\
\frac{4}{3}(1-t) \\
2 t \\
\frac{4}{3} t \\
t
\end{array}\right)=0
$$

The solution of above equation is $t^{*}=\frac{620}{4147} \approx 0.15$.

Step 4. Verify the value of $t^{*}$. Since $t^{*} \notin\left[\frac{1}{4}, \frac{1}{2}\right]$, the value is not valid.

(3) $D_{3}=\left[\frac{1}{2}, \frac{3}{4}\right]$.

Step 1 . Note that the coefficients $a_{i}$ and $b_{i}$ are as follows in this case.

$$
a_{1}=-1, b_{1}=1 ; a_{2}=-\frac{4}{3}, b_{2}=\frac{4}{3} ; a_{3}=-2, b_{3}=2 ; a_{4}=\frac{4}{3}, b_{4}=0 ; a_{5}=1, b_{5}=0
$$

Step 2. Calculate $V$ and $U(t)$. Based on equation (3.9) and equation (3.10), one can calculate $V$ and $U(t)$ as follows.

$$
V^{T}=\left(-\frac{7}{3},-\frac{28}{9},-\frac{14}{3}, \frac{52}{9}, \frac{13}{3}\right) ; \quad(U(t))^{T}=\left(-t+1, \frac{4}{3}(1-t), 2(1-t), \frac{4}{3} t, t\right)
$$

Step 3. Solve the equation (3.12).

$$
\left(-\frac{7}{3},-\frac{28}{9},-\frac{14}{3}, \frac{52}{9}, \frac{13}{3}\right)\left(\begin{array}{ccccc}
1 & -1 & 1 & 2 & -3 \\
-1 & 2 & -3 & -3 & 4 \\
1 & -3 & 5 & 6 & -4 \\
2 & -3 & 6 & 5 & -8 \\
-3 & 4 & -4 & -8 & 16
\end{array}\right)\left(\begin{array}{c}
-t+1 \\
\frac{4}{3}(1-t) \\
2(1-t) \\
\frac{4}{3} t \\
t
\end{array}\right)=0
$$

The solution of above equation is $t^{*}=\frac{662}{595}>1$.

Step 4. Verify the value of $t^{*}$. Since $t^{t *} \notin\left[\frac{1}{2}, \frac{\breve{4}}{4}\right]$, the value is not valid.

(4) $D_{4}=\left[\frac{3}{4}, 1\right]$.

Step 1. Note that the coefficients $a_{i}$ and $b_{i}$ are as follows in this case.

$$
a_{1}=-1, b_{1}=1 ; a_{2}=-\frac{4}{3}, b_{2}=\frac{4}{3} ; a_{3}=-2, b_{3}=2 ; a_{4}=-4, b_{4}=4 ; a_{5}=1, b_{5}=0
$$

Step 2. Calculate $V$ and $U(t)$. Based on equation (3.9) and equation (3.10), one can calculate $V$ and $U(t)$ as follows.

$$
V^{T}=\left(-1,-\frac{4}{3},-2,-4, \frac{25}{3}\right) ; \quad(U(t))^{T}=\left(-t+1, \frac{4}{3}(1-t), 2(1-t), 4(1-t), t\right)
$$

Step 3. Solve the equation (3.12). 


$$
\left(-1,-\frac{4}{3},-2,-4, \frac{25}{3}\right)\left(\begin{array}{ccccc}
1 & -1 & 1 & 2 & -3 \\
-1 & 2 & -3 & -3 & 4 \\
1 & -3 & 5 & 6 & -4 \\
2 & -3 & 6 & 5 & -8 \\
-3 & 4 & -4 & -8 & 16
\end{array}\right)\left(\begin{array}{c}
-t+1 \\
\frac{4}{3}(1-t) \\
2(1-t) \\
4(1-t) \\
t
\end{array}\right)=0
$$

The solution of above equation is $t^{*}=\frac{4354}{5893} \approx 0.74$.

Step 4. Verify the value of $t^{*}$. Since $t^{*} \notin\left[\frac{3}{4}, 1\right]$, the value is not valid.

Step 7. Find the global maximum(minimum) value. Combining the cases (1), (2), (3) and (4) together, one can conclude that this StQP problem has a minimum value

$$
f\left(x^{*}\right)=\frac{193481}{1408969} \approx 0.137 \text { at } x^{*}=\left(\frac{387}{1187}, \frac{384}{1187}, \frac{192}{1187}, \frac{128}{1187}, \frac{96}{1187}\right) .
$$

One can use Lagrangian function method to calculate the KKT point. The solution of Lagrangian function method is

$x^{*}=\left(\frac{128}{339}, \frac{46}{113},-\frac{2}{339}, \frac{58}{339}, \frac{17}{339}\right)$. It is clear that this $x^{*}$ is not a solution of StQP because $x^{*} \notin \Delta$.

Example 5. Solve the following StQP problem

$$
\max _{\mathbf{x} \in \Delta} f(x)=x^{T} Q x
$$

where $x \in R^{5}, \Delta$ is the standard simplex, and $Q$ is as follows.

$$
Q=\left(\begin{array}{lllll}
0 & 1 & 1 & 0 & 0 \\
1 & 0 & 1 & 1 & 0 \\
1 & 1 & 0 & 1 & 0 \\
0 & 1 & 1 & 0 & 1 \\
0 & 0 & 0 & 1 & 0
\end{array}\right)
$$

The symmetric matrix $Q$ is indefinite because it has three negative eigenvalues, and two positive eigenvalues.

A triangle possibility distribution is given by the following five TFNs in $D=[0,1]$.

$$
\left(0,0, \frac{1}{2}\right),\left(0, \frac{1}{4}, \frac{3}{4}\right),\left(0, \frac{1}{2}, 1\right),\left(\frac{1}{4}, \frac{3}{4}, 1\right),\left(\frac{1}{2}, 1,1\right)
$$

The membership functions of TFNs are as follows.

$$
\begin{gathered}
\lambda_{1}(t)=\left\{\begin{array}{ll}
1-2 t & t \in\left[0, \frac{1}{2}\right] \\
0 & t \in\left[\frac{1}{2}, 1\right]
\end{array}, \lambda_{2}(t)= \begin{cases}4 t & t \in\left[0, \frac{1}{4}\right] \\
\frac{3}{2}-2 t & t \in\left[\frac{1}{4}, \frac{3}{4}\right] \\
0 & t \in\left[\frac{3}{4}, 1\right]\end{cases} \right. \\
\lambda_{3}(t)=\left\{\begin{array}{ll}
2 t & t \in\left[0, \frac{1}{2}\right] \\
2(1-t) & t \in\left[\frac{1}{2}, 1\right]
\end{array}, \lambda_{4}(t)= \begin{cases}0 & t \in\left[0, \frac{1}{4}\right] \\
2 t-\frac{1}{2} & t \in\left[\frac{1}{4}, \frac{3}{4}\right] \\
4(1-t) & t \in\left[\frac{3}{4}, 1\right]\end{cases} \right. \\
\lambda_{5}(t)= \begin{cases}0 & t \in\left[0, \frac{1}{2}\right] \\
2 t-1 & t \in\left[\frac{1}{2}, 1\right]\end{cases}
\end{gathered}
$$

The mean values are $d_{1}^{m}=0, d_{2}^{m}=\frac{1}{4}, d_{3}^{m}=\frac{1}{2}, d_{4}^{m}=\frac{3}{4}, d_{5}^{m}=1$, which divides $D=[0,1]$ into four domains, such as $D_{1}=\left[0, \frac{1}{4}\right], D_{2}=\left[\frac{1}{4}, \frac{1}{2}\right], D_{3}=\left[\frac{1}{2}, \frac{3}{4}\right]$ and $D_{4}=\left[\frac{3}{4}, 1\right]$. Let us solve the StQP in each domain. 
(1) $D_{1}=\left[0, \frac{1}{4}\right]$.

Step 1. Note that the coefficients $a_{i}$ and $b_{i}$ are as follows in this case.

$$
a_{1}=-2, b_{1}=1 ; a_{2}=4, b_{2}=0 ; a_{3}=2, b_{3}=0 ; a_{4}=0, b_{4}=0 ; a_{5}=0, b_{5}=0 .
$$

Step 2. Calculate $V$ and $U(t)$. Based on equation (3.9) and equation (3.10), one can calculate $V$ and $U(t)$ as follows.

$$
V^{T}=(-6,4,2,0,0) ; \quad(U(t))^{T}=(-2 t+1,4 t, 2 t, 0,0) .
$$

Step 3. Solve the equation (3.12).

$$
(-6,4,2,0,0)\left(\begin{array}{ccccc}
0 & 1 & 1 & 0 & 0 \\
1 & 0 & 1 & 1 & 0 \\
1 & 1 & 0 & 1 & 0 \\
0 & 1 & 1 & 0 & 1 \\
0 & 0 & 0 & 1 & 0
\end{array}\right)\left(\begin{array}{l}
-2 t+1 \\
4 t \\
2 t \\
0 \\
0
\end{array}\right)=0
$$

The solution of above equation is $t^{*}=\frac{3}{16}$.

Step 4. Verify the value of $t^{*}$. Since $t^{*} \in\left[0, \frac{1}{4}\right]$, the value is valid.

Step 5. Find the values of $x^{*}$ and $f\left(x^{*}\right)$. The value of $x^{*}$ is obtained from equation (3.5).

$$
x^{*}=\left.\left(\frac{1-2 t}{1+4 t}, \frac{4 t}{1+4 t}, \frac{2 t}{1+4 t}, 0,0\right)\right|_{t=\frac{3}{16}}=\left(\frac{5}{14}, \frac{3}{7}, \frac{3}{14}, 0,0\right) \text {.and } f\left(x^{*}\right)=\frac{63}{98} \approx 0.64
$$

Step 6. Determine $f\left(x^{*}\right)$ is a local maximum or minimum value. One can calculate $S(t)$ in equation (3.13) as follows.

$$
S(t)=(-6,4,2,0,0)\left(\begin{array}{ccccc}
0 & 1 & 1 & 0 & 0 \\
1 & 0 & 1 & 1 & 0 \\
1 & 1 & 0 & 1 & 0 \\
0 & 1 & 1 & 0 & 1 \\
0 & 0 & 0 & 1 & 0
\end{array}\right)\left(\begin{array}{l}
-2 \\
4 \\
2 \\
0 \\
0
\end{array}\right)=-32<0
$$

based on Theorem 3.4, $f\left(x^{*}\right)=\frac{63}{98} \approx 0.64$ is a local maximum value.

(2) $D_{2}=\left[\frac{1}{4}, \frac{1}{2}\right]$.

Step 1. Note that the coefficients $a_{i}$ and $b_{i}$ are as follows in this case.

$$
a_{1}=-2, b_{1}=1 ; a_{2}=-2, b_{2}=\frac{3}{2} ; a_{3}=2, b_{3}=0 ; a_{4}=2, b_{4}=-\frac{1}{2} ; a_{5}=0, b_{5}=0
$$

Step 2. Calculate $V$ and $U(t)$. Based on equation (3.9) and equation (3.10), one can calculate $V$ and $U(t)$ as follows.

$$
V^{T}=(-4,-4,4,4,0) ; \quad(U(t))^{T}=\left(1-2 t, \frac{3}{2}-2 t, 2 t, 2 t-\frac{1}{2}, 0\right)
$$

Step 3. Solve the equation (3.12).

$$
(-4,-4,4,4,0)\left(\begin{array}{ccccc}
0 & 1 & 1 & 0 & 0 \\
1 & 0 & 1 & 1 & 0 \\
1 & 1 & 0 & 1 & 0 \\
0 & 1 & 1 & 0 & 1 \\
0 & 0 & 0 & 1 & 0
\end{array}\right)\left(\begin{array}{c}
1-2 t \\
\frac{3}{2}-2 t \\
2 t \\
2 t-\frac{1}{2} \\
0
\end{array}\right)=0
$$

The solution of above equation is $t^{*}=\frac{3}{8}=0.375$. 
Step 4. Verify the value of $t^{*}$. Since $t^{*} \in\left[\frac{1}{4}, \frac{1}{2}\right]$, the value is valid.

Step 5. Find the values of $x^{*}$ and $f\left(x^{*}\right)$. The value of $x^{*}$ is obtained from equation (3.5).

$$
x^{*}=\left.\left(\frac{1-2 t}{2}, \frac{\frac{3}{2}-2 t}{2}, \frac{2 t}{2}, \frac{2 t-\frac{1}{2}}{2}, 0\right)\right|_{t=\frac{3}{8}}=\left(\frac{1}{8}, \frac{3}{8}, \frac{3}{8}, \frac{1}{8}, 0\right) \text {.and } f\left(x^{*}\right)=\frac{21}{32} \approx 0.66
$$

Step 6. Determine $f\left(x^{*}\right)$ is a local maximum or minimum value. One can calculate $S(t)$ in equation (3.13) as follows.

$$
S(t)=(-4,-4,4,4,0)\left(\begin{array}{ccccc}
0 & 1 & 1 & 0 & 0 \\
1 & 0 & 1 & 1 & 0 \\
1 & 1 & 0 & 1 & 0 \\
0 & 1 & 1 & 0 & 1 \\
0 & 0 & 0 & 1 & 0
\end{array}\right)\left(\begin{array}{l}
-2 \\
-2 \\
2 \\
2 \\
0
\end{array}\right)=-16<0
$$

based on Theorem 3.4, $f\left(x^{*}\right)=\frac{21}{32} \approx 0.66$ is a local maximum value.

(3) $D_{3}=\left[\frac{1}{2}, \frac{3}{4}\right]$.

Step 1 . Note that the coefficients $a_{i}$ and $b_{i}$ are as follows in this case.

$$
a_{1}=0, b_{1}=0 ; a_{2}=-2, b_{2}=\frac{3}{2} ; a_{3}=-2, b_{3}=2 ; a_{4}=2, b_{4}=-\frac{1}{2} ; a_{5}=2, b_{5}=-1
$$

Step 2. Calculate $V$ and $U(t)$. Based on equation (3.9) and equation (3.10), one can calculate $V$ and $U(t)$ as follows.

$$
V^{T}=(0,-4,-4,4,4) ; \quad(U(t))^{T}=\left(0, \frac{3}{2}-2 t, 2-2 t, 2 t-\frac{1}{2}, 2 t-1\right)
$$

Step 3. Solve the equation (3.12).

$$
(0,-4,-4,4,4)\left(\begin{array}{lllll}
0 & 1 & 1 & 0 & 0 \\
1 & 0 & 1 & 1 & 0 \\
1 & 1 & 0 & 1 & 0 \\
0 & 1 & 1 & 0 & 1 \\
0 & 0 & 0 & 1 & 0
\end{array}\right)\left(\begin{array}{c}
0 \\
\frac{3}{2}-2 t \\
2-2 t \\
2 t-\frac{1}{2} \\
2 t-1
\end{array}\right)=0
$$

The solution of above equation does not exist. That is, when $t \in D_{3}=\left[\frac{1}{2}, \frac{3}{4}\right]$ there is no solution.

(4) $D_{4}=\left[\frac{3}{4}, 1\right]$.

Step 1. Note that the coefficients $a_{i}$ and $b_{i}$ are as follows in this case.

$$
a_{1}=0, b_{1}=0 ; a_{2}=0, b_{2}=0 ; a_{3}=-2, b_{3}=2 ; a_{4}=-4, b_{4}=4 ; a_{5}=2, b_{5}=-1 .
$$

Step 2. Calculate $V$ and $U(t)$. Based on equation (3.9) and equation (3.10), one can calculate $V$ and $U(t)$ as follows.

$$
V^{T}=(0,0,-2,-4,6) ; \quad(U(t))^{T}=(0,0,2-2 t, 4-4 t, 2 t-1) .
$$

Step 3. Solve the equation (3.12).

$$
(0,0,-2,-4,6)\left(\begin{array}{ccccc}
0 & 1 & 1 & 0 & 0 \\
1 & 0 & 1 & 1 & 0 \\
1 & 1 & 0 & 1 & 0 \\
0 & 1 & 1 & 0 & 1 \\
0 & 0 & 0 & 1 & 0
\end{array}\right)\left(\begin{array}{l}
0 \\
0 \\
2-2 t \\
4-4 t \\
2 t-1
\end{array}\right)=0
$$

The solution of above equation is $t^{*}=\frac{3}{4}=0.75$. 
Step 4. Verify the value of $t^{*}$. Since $t^{*} \in\left[\frac{3}{4}, 1\right]$, the value is valid.

Step 5. Find the values of $x^{*}$ and $f\left(x^{*}\right)$. The value of $x^{*}$ is obtained from equation (3.5).

$$
x^{*}=\left.\left(\frac{0}{5-4 t}, \frac{0}{5-4 t}, \frac{4-4 t}{5-4 t}, \frac{2 t-1}{5-4 t}\right)\right|_{t=\frac{3}{4}}=\left(0,0, \frac{1}{4}, \frac{1}{2}, \frac{1}{4}\right) \text {.and } f\left(x^{*}\right)=\frac{1}{2}=0.5
$$

Step 6. Determine $f\left(x^{*}\right)$ is a local maximum or minimum value. One can calculate $S(t)$ in equation (3.13) as follows.

$$
S(t)=(0,0,-2,-4,6)\left(\begin{array}{lllll}
0 & 1 & 1 & 0 & 0 \\
1 & 0 & 1 & 1 & 0 \\
1 & 1 & 0 & 1 & 0 \\
0 & 1 & 1 & 0 & 1 \\
0 & 0 & 0 & 1 & 0
\end{array}\right)\left(\begin{array}{l}
0 \\
0 \\
-2 \\
-4 \\
2
\end{array}\right)=-16<0
$$

Based on Theorem 3.4, $f\left(x^{*}\right)=\frac{1}{2}=0.5$ is a local maximum value.

Step 7. Find the global maximum(minimum) value. Combining the cases (1), (2), (3) and (4) together, one can conclude that this StQP has a global maximum value $f\left(x^{*}\right)=\frac{21}{32}$ at point $x^{*}=\left(\frac{1}{8}, \frac{3}{8}, \frac{3}{8}, \frac{1}{8}, 0\right)$.

One can use Lagrangian function method to calculate the KKT point. The solution of Lagrangian function method is $x^{*}=\left(-\frac{1}{3}, \frac{1}{3}, \frac{1}{3}, \frac{2}{3}, 0\right)$. It is clear that this $x^{*}$ is not a solution of StQP because $x^{*} \notin \Delta$.

\section{The Analysis of Complexity}

Note that if matrix Q is indefinite, the StQP problem is NP-hard (Bomze, 2002). Even if matrix Q has only one negative eigenvalue, the StQP problem is NP-hard. It is clear that the symmetric matrices $Q$ in the examples in section 4 are indefinite because they have negative eigenvalue(s).

The algorithm shows that solving $n+1$ ( $n$ is the number of variables) number of linear equations is able to solve StQP problems. Note that solving $n+1$ number of linear equations is in P. We claim that StQP problems can be solved in polynomial time.

\section{Theorem 5.1 StQP problems are in $P$.}

Proof. Note that solving $n+1$ number of linear equations is in P. According to Theorem 3.3, solving StQP is equivalent to solving $n+1$ number of linear equations. Therefore, StQP problems are in $\mathrm{P}$.

Fortnow describes that (1) finding Nash Equilibriums with specific properties in a number of environments is NP-complete and (2) an efficient solution to any NP-complete problem would imply $P=N P$ (Fortnow, 2009). The proposed algorithm indicates that NP-hard problem, StQP problem, is in $P$.

The algorithm can be applied to multiple variables quadratic optimization problems. In fact, paper (Gao, 2015) can be considered an application of the algorithm to two player games for calculating Nash equilibrium in game theory.

\section{Conclusion}

The proposed algorithm uses the possibility distribution functions. The benefits of using possibility distribution functions are, first the constrained conditions of the standard simplex are encapsulated into the possibility distribution functions, secondly the application of possibility distribution gives an approximation formula of StQP problems by mapping variable $x \in R^{n}$ into the possibility distribution.

The proposed algorithm does not require iterative method (Bomze, 1998) to find the global maximum(minimum) solution of StQP problems. It finds StQP solutions by solving a set of linear equations regardless whether the symmetric matrix $Q$ is indefinite or not. The algorithm reduced the computational complexity of NP-hard StQP problems into P problems.

The proposed algorithm does not only find KKT points, but it can also identify that the optimal values are either local maximum or local minimum values. The examples demonstrate the feasibility and efficiency of the algorithm. Example 3 indicates that the algorithm is able to find out both the maximum value and the minimum 
value for a StQP problem.

When one knows the probability distribution over the standard simplex, by using the probability-possibility transformation theory to define the associated possibility distribution, then one can find the solutions of StQP problems in polynomial time by using the algorithm.

Further research may apply this algorithm to solve practical problems, and compare the proposed algorithm with other methods, for example, gradient projection method, branch and bound approach, to discover the differences.

\section{References}

Ammear, E., \& Khalifa, H. A. (2003). Fuzzy Portfolio Optimization a Quadratic Programming Approach. Chaos, Solitons and Fractals, 18, 1045-1054.

Bolor, J. (2013). Indefinite Copositive Matrices with Exactly One Positive Eigenvalue or Exactly One Negative Eigenvalue. Electronic Journal of Linear Algebra, 26, 754-761. https://doi.org/10.13001/1081-3810.1685

Bomze, I. M. (1998). On Standard Quadratic Optimization Problems. Journal of Global Optimization, 13, 369-387.

Bomze, I. M. (2002). Branch-and-Bound Approaches to Standard Quadratic Optimization Problems. Journal of Global Optimization, 22, 17-37.

Chen, X., Peng, J., \& Zhang, S. (2013). Sparse Solutions to random Standard Quadratic Optimization Problems. Math. Program., Ser. A, 141, 273-293.

Drakopoulos, J. (1995). Probabilities, Possibilities, and Fuzzy Sets. Fuzzy Sets and Systems, 75(1), 1-15.

Dubois, D., Foulloy, L., Mauris, G., \& Prade, H. (2004). Probability-Possibility Transformations, Triangular Fuzzy Sets, and Probabilistic Inequalities. Reliable Computing, 10, 273-297.

Fortnow, L. (2009). The Status of the P Versus NP Problem. Communications of the ACM, 52(9), 78-86. Retrieved from http://dl.acm.org/citation.cfm?doid=1562164.1562186

Gao, L. (2015). An Efficient Algorithm for Finding Mixed Nash Equilibrium in 2-Player Games. Journal of Game Theory, 4(3), 45-55. Retrieved from http://article.sapub.org/10.5923.j.jgt.20150403.01.html

Kaufmann, A., \& Gupta, M. M. (1998). Fuzzy mathematical models in engineering and management Science, Elsevier, Amsterdam.

Kozlov, M. K., Tarasov, S. P., \& Haijan, L. G. (1979). Polynomial Solvability of Convex Quadratic Programming. Soviet Math. Doklady 20.

L. A. Zadeh. (1978). Fuzzy Sets as a Basis for a Theory of Possibility. Fuzzy Sets and Systems, 1, 3-28.

L. A. Zadeh. (2014). A Note on Similarity-based Definitions of Possibility and Probability. Information Sciences, 267, 334-336. https://doi.org/10.1016/j.ins.2014.01.046

Liu, S. T. (2007). Quadratic Programming with Fuzzy Parameters: A Membership Function Approach. Chaos, Solitons and Fractals.

Liu, S. T. (2007). Solving Quadratic Programming with Fuzzy Parameters Based on Extension Principle. The Proceeding of FUZZ-IEEE 2007, 1-5.

Ohlson, M., \& Koski, T. (2012). On the Distribution of Matrix Quadratic Form Communications in Statistics-Theory and Methods, 18(41), 3403-3415

Serrurier, M., \& Prade, H. (2.13). An Information Distance for Estimating the Faithfulness of a Probability Distribution, Viewed as a Family of Probability Distributions, with Respect to Data. International Journal of Approximate Reasoning, 54(7), 919-933. https://doi.org/10.1016/j.ijar.2013.01.001

Silva, R. C., Cruz, C., Verdegay, J. L., \& Yamakami, A. (2010). A Survey of Fuzzy Convex Programming Models. Fuzzy Optimization, Studfuzz, 254, 127-143. Springer-Verlag.

Singpurwalla, D. N., \& Booker, M. J. (2004). Membership Functions and Probability Measures of Fuzzy Sets. Journal of the American statistical Association, 99(467). https://doi.org/10.1198/016214504000001196

Tang, J., \& Wang, D. (1997). A Nonsymmetric Model for Fuzzy Nonlinear Programming Problems with Penalty Coefficients. Computers and Operations Research, 24(8), 717-725.

Tang, J., \& Wang, D. (1997). An Interactive Approach Based on a Genetic Algorithm for a Type of Quadratic Programming Problems with Fuzzy Objective and Resources. Computers and Operations Research, 24(5), 
413-422.

Tonon, F., \& Bernardini, A. (1998). A Random Set Approach to the Optimization of Uncertain Structures. Computers and Structures, 68, 583-600.

\section{Copyrights}

Copyright for this article is retained by the author(s), with first publication rights granted to the journal.

This is an open-access article distributed under the terms and conditions of the Creative Commons Attribution license (http://creativecommons.org/licenses/by/4.0/). 